\title{
Congestion Control Reliability and Adaptive Cooperation for Efficient Underwater Sensor Networks
}

\author{
Junaid Ahmed Inam ${ }^{1},{ }^{2}$, Sheeraz Ahmed ${ }^{2}$, Najeeb Ullah ${ }^{1}$, Ali Raza ${ }^{1},{ }^{2}$, Ata-ur-Rehman ${ }^{1},{ }^{2}$, Asghar \\ Khattak $^{1},{ }^{2}$, Saqib Shahid Rahim ${ }^{2,3}$ \\ ${ }^{1}$ CECOS University Peshawar, Pakistan ${ }^{2}$ Career Dynamics Research Centre, Peshawar, Pakistan ${ }^{3}$ ABASYN University \\ Peshawar, Pakistan
}

\section{Abstract}

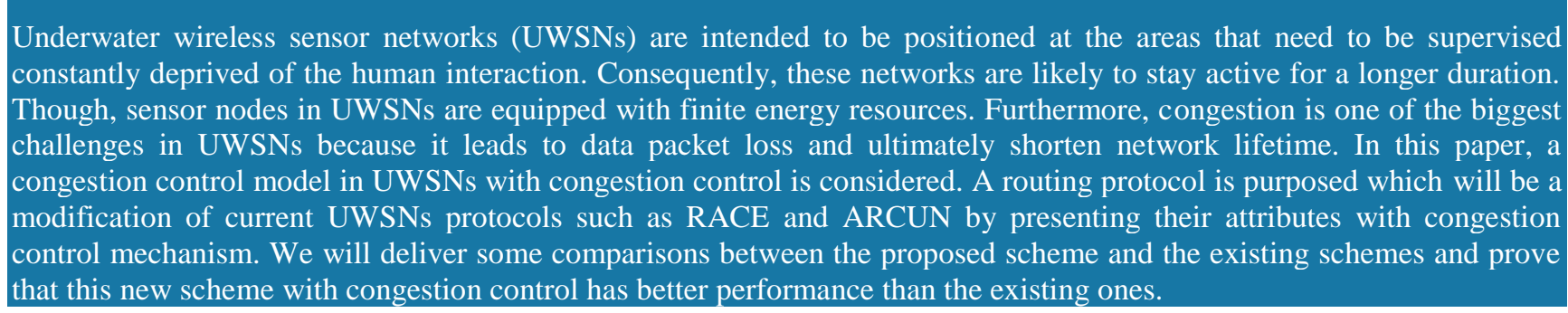

Keywords: Stability Period, UWSN, Throughput, Transmission loss.

Received on 17 December 2017, accepted on 16 February 2018, published on 13 April 2018

Copyright (C) 2018 Junaid Ahmed Inam et al., licensed to EAI. This is an open access article distributed under the terms of the Creative Commons Attribution licence (http://creativecommons.org/licenses/by/3.0/), which permits unlimited use, distribution and reproduction in any medium so long as the original work is properly cited.

doi: 10.4108/10.4108/eai.13-4-2018.154470

\section{Introduction}

Technologies like Wireless Sensor Networks (WSNs) are recognized by various industries as an economical alternate to currently implemented and expensive approaches used in technology [1]. Terrestrial communication is a standard system of WSN which uses radio waves. WSNs currently uses many new techniques for better communication. These techniques are also tested by researchers in the field of underwater [2]. The scope for various innovative networking structures have been opened by key technological developments in associated fields. Approximately seventy percent of the earth surface is roofed by water. This is generally unmapped region which make humans interested to discover it. Usual or semisynthetic catastrophes have taken place over the years which provoked vital attention in observing marine environment. Underwater Wireless Sensor Networks (UWSNs) forms an optimistic innovation for empowering and improving a few key applications in underwater exploration. UWSNs have established many applications as they are used in sea exploration, data collection, pollution monitoring, tactical surveillance, disaster prevention, military applications and mine surveys [3]. Transmission of data from sensor nodes to one of the sinks at sea surface using multi hop is required by underwater applications. The structure of UWSNs is presented in figure 1. It consists of small sized sensors fixed to the bottom of the sea which are connected to each other by wireless acoustic channel. Data is transmitted from sensor nodes to Autonomous Underwater Vehicles (AUVs) which dive inside the water and a surface sink, available at water surface assembled with radio and acoustic channels. These sensor nodes inspect native under water events and send their reports using multiple hops to one of the sinks at the water surface using acoustic signals [4]. The surface sink is equipped with acoustic communication sensors and satellite transmitter to communicate with the onshore station, thus assembles data from the surface sink and handle UWSNs. 


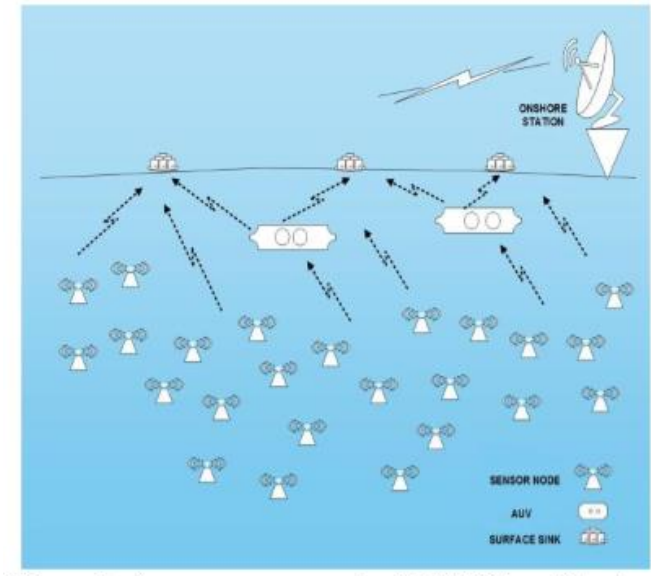

Figure 1: A common scenario of UWSN architecture

Underwater sensor nodes are designed to perform consistently over long periods of time without repairing, servicing, or recharging in the tough isolated environment of the deep sea. These nodes must be deployed in a spatial arrangement due to their inability of autonomous lateral movement in underwater networks [5]. UWSNs need to reconfigure a communication path through a continuous route that is separate from the previous path by which communication was made [6]. The underwater environment is much different from terrestrial communications due to the high dense salty water, electromagnetic, scattering and absorption effects and optical signals which cannot be transmitted due to high attenuation [7]. Current communication protocols for terrestrial networks do not work under water due to low bandwidth and large latency. These technologies are still expensive as compared to terrestrial networks. Deployment of sensor nodes are sparser in these networks and on the other hand terrestrial networks are densely deployed. Nodes mobility can be projected easily in terrestrial but the prediction of node mobility is difficult in underwater networks. Due to large gaps between sensors nodes, reading taken from underwater networks are not correlated compared to terrestrial WSNs [1]. Congestion Control In UWSNs, sensor nodes are usually idle but when they detect an event, they start generating and transmitting data to one or more surface sinks. Congestion may occurs due to sudden increase in network load at sink and the sensor nodes around it depending on the transmission rates, which results in packet losses and low network performance. In UWSNs, congestion is elevated further due to bad channel conditions. This issue stimulate the requirement for congestion control schemes in UWSNs [1]. Congestion control incorporate measures taken for manipulating the data transmission within the network in order to encounter congestion and avoid congestion failure [4]. Most of congestion control schemes assumed that major network losses are due to congestion only which cause more problems in UWSNs. TCP based protocols cannot be suitable for UWSNs due to their failure to differentiate between packet losses due to congestion and those due to channel error [8]. The generic reasons for congestion are buffer overflow, lack of synchronization and many to one nature of the network (sources to a common sink) [1].

\section{Related Work}

The research in [1] presented numerous major features and structures of UWSNs. The researchers highlighted the emerging research issues and challenges imposed by UWSNs. Evaluation of the research issues in UWSNs was done with respect to the physical layer, data link layer, network layer, transport layer and application layer. In [2], the researchers proposed energy efficient physical layer cooperation for the design of a network layer routing scheme for UWSNs using reliability as a vital aspect. Network nodes cooperatively performed their transmission of data strengthen by a unidirectional antenna which save nodes energy. At physical layer, diverse cooperation and at network layer hop to hop routing, minimized the routing energy due to combined optimization. Simulation results showed that RACE routing scheme achieves improved packet delivery ratio, high stability period, less consumption of energy and low end-to-end delay as compared to AMCTD and ACE. In [3] the authors proposed a reliable, energy-efficient, and high throughput routing protocol Cooperative UWSN (Co-UWSN) for UWSNs to improve the performance of network. Distance and signal-to-noise ratio computation of the channel conditions are utilized by destination and relays nodes as cost functions. This helped in transferring of data with much reduced path loss. Simulation results indicated better performance of Co-UWSN protocol in terms of end-to-end delay, energy consumption, and network lifetime when compared to protocols like EEDBR, iAMCTD and Coop (Re and dth). The authors have presented an energy-efficient and high-throughput cooperative transmission scheme, ARCUN for UWSNs in [4] to improve the network performance. Relays nodes applied signal-to-noise ratio and distance computation of the underwater channel. Optimal role of cooperation provided load balancing, network stability period and packet delivery ratio in the network. The research in [5] proposed a comparative analysis of UWSN routing protocols over real time multimedia data to extend their in-built support for transmission of real time data traffic. The overall performance traits concerning these protocols have been studied under different multimedia load conditions with variable mobility which explored applicability of congestion control protocols in UWSNs. In [6] the researchers adopted a top-down approach to explore the research challenges in mobile UWSN design. Along the layered protocol stack, the authors proceed roughly from the top application layer to the bottom physical layer. At each layer, a set of new design intricacies was studied. The conclusion was that building scalable mobile UWSNs is a challenge that must be 
answered by interdisciplinary efforts of acoustic communications, signal processing, and mobile acoustic network protocol design. The authors in [7] examined the key methodologies and challenges in the design and implementation of certain underwater routing protocols like VBF, DBR, H2-DAB, and QELAR etc. in terms of their localization techniques, energy minimization characteristics, holding time calculations, achievement of robustness and scalability. These protocols play an important role in the ongoing research on routing layer in UWSNs. The writers proposed a biologically-inspired congestion control protocol for UWSNs in [8] that captured the effects of packet losses due to congestion and high link error rates, detected shadow zones and improved the throughput of network transmission which consequently, stabilizes the system. The authors in [9] presented a data aggregation scheme to generate an effective aggregative route for the UWSNs using multiple parameters including distance, energy, residual energy and congestion. The scheme originated a chain based path over a less congested nodes so that network life would be improved and it used an algorithmic approach to select the next neighboring node. In [10] the authors suggested the combination of acoustics and optics technologies to maximize their advantages using SDN architecture in which acoustic channel served as the control plane for issuing different instructions to AUVs, and the optical channel served as the data plane for fast transfer of data. The authors purposed the optimization of a congestionaware signaling aggregation scheme in [11] based on cellular network, which was designed considering several requirements of UASNs services and the congestion situation in the LTE network. Simulations of the scheme showed improved system efficiency and significant elimination of signaling congestion, particularly for the emergency UASN service. In [12] the authors projected a congestion control model in UWSNs with time delay as a bifurcating parameter to investigate the boundedness of the positive equilibrium to keep the samples density of sensor node in the environmental carrying capacity. UWSNs would be congested and bifurcation would occurred when the communication time delay passed a critical value. The authors applied the normal form theory and the center manifold theorem to derived solutions for the stability of network.

\section{Problem Statement}

According to the literature, preponderance of researchers [21],[22] used depth of the sensor nodes as a metric for forwarding data packets but there subsists lack of proper load balancing and by this uneven distribution of load of motes. Hence efficient energy consumption is not appropriately reduced by inappropriate balancing of load among UWSNs motes. [21] takes the only parameter that is depth of motes to forward data. DBR tries to achieve lengthier lifetime of UWSN however the stability period finishes soon by redundant forwarding of data and abundant load on motes that have low depth in the network. [22] technique is based on receiver in which every mote that has lesser depth selects as forwarder. However redundancy of packets during the transmission takes much energy. EEDBR not a cooperative scheme hence packets are sent from source motes to endpoint using an only link but in multi hop manner. By noise and multipath fading environ of UWSN, most of the time the signals undergoes high bit error rate (BER). To overcome the limitation in above discussion we proposed DAE scheme for UWSNs. In this scheme, the performance is increased by taking care of delivery ratio, stability period and end-end-delay.

There are many protocols which are recommended for terrestrial networks but they cannot be used for UWSNs due to challenges like propagation delay, high bit error rate and limited bandwidth (i.e. $<100 \mathrm{kHz}$ ) so all the sensor nodes are equipped with a low bandwidth acoustic modem therefore its propagation latency is five times higher than the radio channel i.e. sensor nodes can propagate upto 1 to $3 \mathrm{~m} / \mathrm{sec}$ and they are generally considered as static [3]. Underwater nodes utilize more power and replacement of nodes or batteries is very difficult [7]. Current technology for conventional underwater exploration is not suitable for applications covering a large area so localized exploration is more suitable than remote exploration as it only depend on a single expensive underwater un-manned device or a limited underwater network. UWSNs acquires high cost for nodes deployment, maintenance and recovery due to unpredictable undersea conditions [6].All these restrictions create packet collisions which make UWSN vulnerable to congestion.

In UWSNs the failure of communication channel highly affects its reliability. In order to ensure the network reliability the authors in [2] and [4] used reliability as performance matric for end-to-end transmission from source node to destination node in UWSNs but do not include any mechanism to control network congestion. Better results could be generated if these authors also considered congestion control techniques to satisfy the challenges of UWSNs.

We want to design a routing protocol which will be a modification of RACE [2] and ARCUN [4] by addressing their features with congestion control mechanism. We will provide some comparisons between the proposed scheme and the existing schemes and show that this new scheme with congestion control has better performance than the existing ones.

\section{Aims and Objective}

Congestion turns out to be a vital issue in UWSNs. Packet loss due to congestion cannot be abolished but can be diminished. Aims and objectives of this research work are following as:

-The aim is to handle UWSNs in an efficient way concerning longer life time while maintaining the quality of service. 
-To minimize the compound multi hops in UWSNs and sustain a low average energy consumption of sensor nodes.

-To avoid the UWSN from a congestive breakdown and maintain congestion free environment.

-To maintain network connectivity for effective routing by distinguishing between good and bad communications links.

-To address the issue of buffer overflow in UWSNs.

\section{Working Methodology}

In the first step of research methodology, I shall conduct literature review of existing routing techniques of UWSNs from cooperation perspective which will help in the clarification of all related concepts. Those reference papers will be selected which will be relevant to my thesis topic. A comparative analysis of existing techniques will be done to proceed in the right direction. This will be only possible if we can have a reproduction of results of existing techniques. This helps in better understanding of the mathematical modeling and simulations conducted by the research community with respect to energy effeciency and network management.Identification of drawbacks of existing techniques will be the next and most crucial step as on the basis of this I can go for the solution of issues which have not been addressed till yet in the field of UWSNs. Mathematical formulation of existing schemes will be done as the next step. The referenced mathematical modeling by research community in older papers will help cover those areas as well which have not been yet addressed.In order to validate the mathematical modeling for the proposed scheme, simulations for the scheme will be conducted using MATLAB as the simulator which is a quite suitable simulator to validate mathematical formulations and in order to check the performance of our proposed scheme and validate its efficiency, a comparison of the scheme will be done with the existing ones through these experiments and simulations. Final step will be the thesis write-up and presented to the department for review of external examiners.

\section{Research Methodology}

In UWSNs several sensor nodes are arbitrarily positioned for monitoring purpose. Sensor nodes and relay nodes are deployed in water at various depths and sink nodes are positioned on the water surface. It is assumed that the nodes do not modify their depth and horizontal mobility of nodes is also prevented. The flowchart of proposed scheme in UWSN is shown in Figure 2, where sensor nodes and forward data to relay node through the acoustic channel. Relay node receives the data and forward it to the succeeding level to the sink (surface station) through the acoustic link. In case of buffer overflow due to congestion i.e. data received very rapidly or bad communication link, some event samples send by sensor nodes must be eliminated at rely node in order to uphold the equilibrium state. The proposed congestion control scheme will be able to encounter congestion by taking measures for manipulating packets transmission to avoid overflow of nodes.

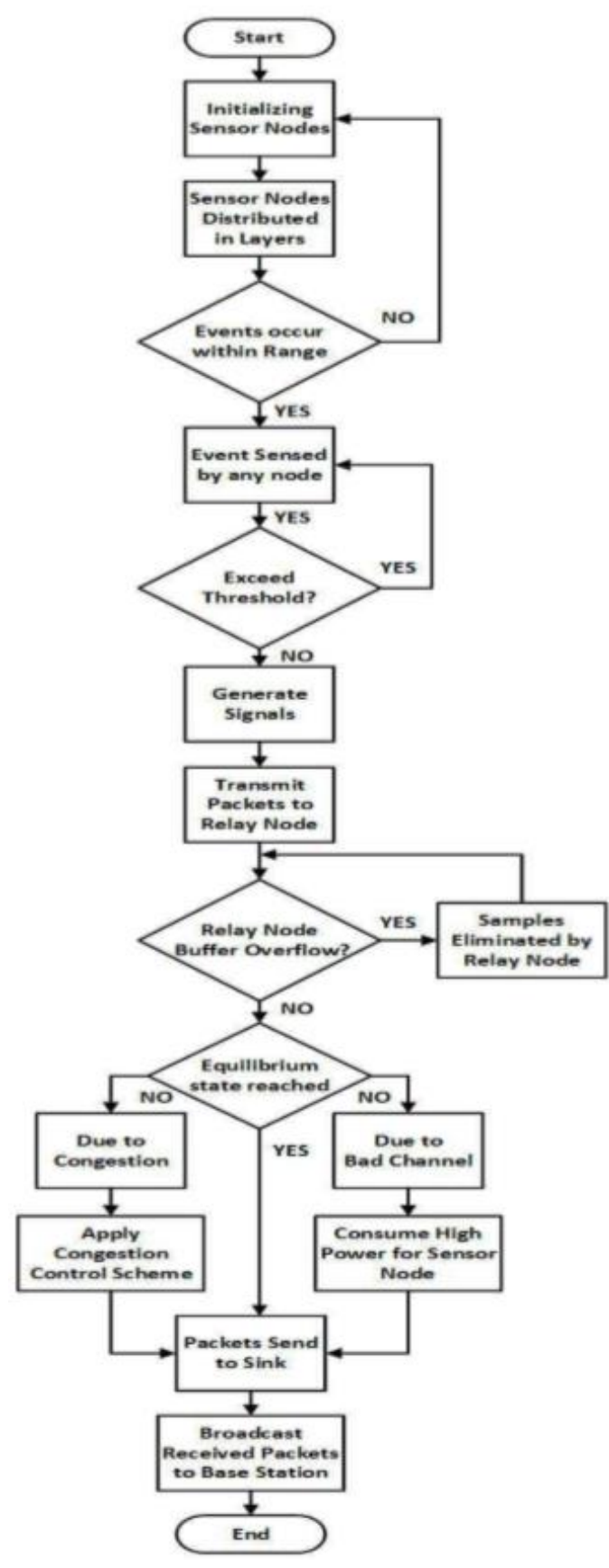

7 Performance Evaluation 
To evaluate the performance of congestion control, we compared CC-RACE scheme that contains the congestion control with the RACE scheme in which congestion control is absent. Model of multiple-sink using conventional methods having 5 surface sinks at surface with 225 arbitrarily deployed motes in $500 \mathrm{~m}$ x $500 \mathrm{~m}$ network area. Range of transmission for the each sensor is supposed to $100 \mathrm{~m}$.

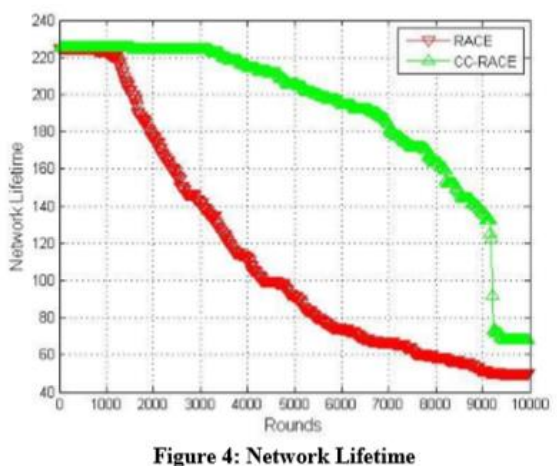

Figure 4: Network Lifetime

\subsection{Transmission Loss}

Figure 5 showing the transmission loss of CC-RACE as well as RACE. The outcome depicts that the transmission loss in CC-RACE is enough smaller than the RACE. In our designed scheme there is no transmission loss till round 3100 while in RACE the transmission loss starts from round 1200 and then this loss is increases very speedily as compare to CC-RACE scheme.

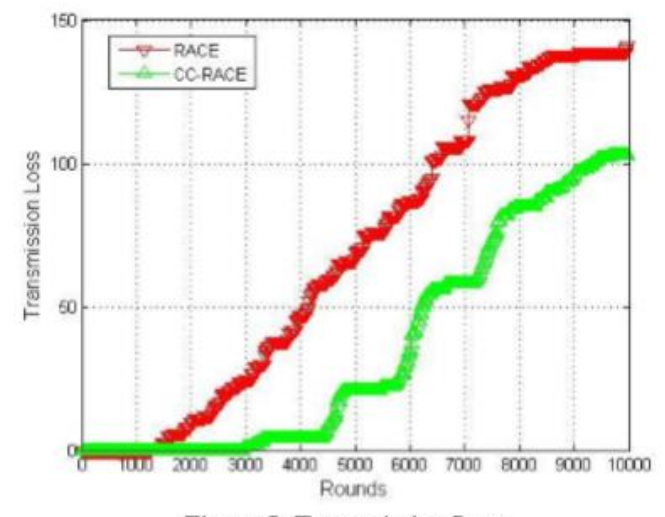

Figure 5: Transmission Loss

\section{References}

1. Manjula, R. B., and Sunilkumar S. Manvi. "Issues in underwater acoustic sensor networks." International Journal of Computer and Electrical Engineering 3, no. 1 (2011): 101.

2. Ahmed, Sheeraz, Nadeem Javaid, Saqib Shahid, Fazle Hadi, and M. Qaiser Azeem. "RACE: Reliability and adaptive cooperation for efficient underwater sensor networks." In Open Source Systems \& Technologies (ICOSST), 2016 International Conference on, pp. 122-128. IEEE, 2016.

3. Ahmed, Shiraz, N. Javaid, F. A. Khan, M. Y. Durrani, A. Ali, A. Shaukat, M. M. Sandhu, Z. A. Khan, and U. Qasim. "Co-UWSN: cooperative energy-efficient protocol for underwater WSNs." International Journal of Distributed Sensor Networks 11, no. 4 (2015): 891410.

4. Ahmed, Sheeraz, Mariam Akbar, Rehmat Ullah, Samin Ahmed, Mohsin Raza, Zahoor Ali Khan, Umar Qasim, and Nadeem Javaid. "ARCUN: Analytical Approach towards 
Reliability with Cooperation for Underwater WSNs." Procedia Computer Science 52 (2015): 576-583.

5. Jain, Shalini, Emmanuel S. Pilli, M. C. Govil, and D. Vijay

Rao. "Performance evaluation of congestion-aware routing protocols for underwater sensor networks with multimedia data." In Underwater Technology (UT), 2015 IEEE, pp. 16. IEEE, 2015.

6. Cui, Jun-Hong, Jiejun Kong, Mario Gerla, and Shengli Zhou. "The challenges of building mobile underwater wireless networks for aquatic applications." Ieee Network 20, no. 3 (2006): 12-18.

7. Ahmed, S., I. U. Khan, Muhammad Babar Rasheed, Manzoor Ilahi, R. D. Khan, Safdar Hussain Bouk, and Nadeem Javaid. "Comparative analysis of routing protocols for under water wireless sensor networks." arXiv preprint arXiv:1306.1148 (2013).

8. Domingo, Mari Carmen. "Marine communities based congestion control in underwater wireless sensor networks." Information Sciences 228 (2013): 203-221.

9. Tomar, Sanjiv Kumar. "A Parametric Chain based Routing Approach for Underwater Sensor Network." arXiv preprint arXiv:1402.5748 (2014)

10. Ruolin Fan, Ciaran Mcgoldrick, and Mario Gerla. "An SDN Architecture for Underwater Search and Surveillance." WONS 20171570316740.

11. Xu, Fangmin, Rong Li, Chenglin Zhao, Haipeng Yao, and Jundong Zhang. "Congestion-aware signaling aggregation scheme for cellular based Underwater Acoustic Sensor Network." In Communication Workshop (ICCW), 2015 IEEE International Conference on, pp. 2528-2533. IEEE, 2015.

12. Dong, Tao, Wenjie Hu, and Xiaofeng Liao. "Dynamics of the congestion control model in underwater wireless sensor networks with time delay." Chaos, Solitons \& Fractals 92 (2016): 130-136. 